\title{
Coordinated Path Following for Mobile Robots
}

\author{
Kiattisin Kanjanawanishkul, Marius Hofmeister, and Andreas Zell \\ University of Tübingen, Department of Computer Science, Sand 1, 72076 Tübingen
}

\begin{abstract}
A control strategy for coordinated path following of multiple mobile robots is presented in this paper. A virtual vehicle concept is combined with a path following approach to achieve formation tasks. Our formation controller is proposed for the kinematic model of unicycle-type mobile robots. It is designed in such a way that the path derivative is employed as an additional control input to synchronize the robot's motion with neighboring robots. A second-order consensus algorithm under undirected information exchange is introduced to derive the control law for synchronization. Our controller was validated by simulations and experiments with three unicycle-type mobile robots.
\end{abstract}

\section{Introduction}

Compared with a single mobile robot, multi-robot systems (MRS) offer many advantages, such as flexibility of operating the group of robots and failure tolerance due to redundancy. Coordination and formation control has been a popular topic of study in multi-robot systems [1]. The problem is defined as the coordination of multiple mobile robots to follow given references and to maintain a desired spatial formation. In the literature, there have been roughly three strategies to formation control of multiple robots: leader-following [2], virtual structure [3], and behavior-based [4]. Each approach has its own advantages and disadvantages. In this paper, we develop a control law based on a virtual vehicle approach for coordinated path following of a group of $N$ mobile robots. The controller is designed in such a way that the derivative of the path parameter is used as an additional control input to synchronize the formation motion. This coordinated path-following problem can be divided into two subproblems, i.e., the path following control problem and the coordination problem. In the first subproblem, a desired geometric path is parameterized by the curvilinear abscissa $s(t) \in \mathbb{R}$ and a path following controller should look at (i) the distance from the robot to the path and (ii) the angle between the robot's velocity vector and the tangent to the path, and then reduce both to zero, without any consideration in temporal specifications. Pioneering work in this area can be found in [5] and references therein. In contrast to trajectory tracking, we have the freedom to select a temporal specification for $s(t)$ in path following. The reference point on the path is the location of a so-called virtual vehicle. The path derivative can be considered as an additional control input, as seen in $[6,7]$.

In the second subproblem, the robot's motion has to be synchronized with its neighbors in order to achieve a desired formation configuration. In [8], synchronized path following is solved by using passivity-based designs and is validated by 
simulation with marine surface vessels. Ghabcheloo, et al. [9] derive the control law for autonomous underwater vehicles (AUVs). Some approaches for coordinated path following control of multiple wheeled mobile robots are proposed in $[10,11]$. In this work, we derive our control law based on a Lyapunov function candidate and a consensus algorithm for a simplified kinematic model of mobile robots. Both path errors and coordination errors are considered in the Lyapunov function and the path parameter is used to synchronize coordination motions via a second-order consensus protocol with a reference velocity.

\section{Problem Statement}

We consider a group of $N$ mobile robots, each of which has the following kinematic equations

$$
\left[\begin{array}{c}
\dot{x}_{i} \\
\dot{y}_{i} \\
\dot{\theta}_{i}
\end{array}\right]=\left[\begin{array}{c}
v_{i} \cos \theta_{i} \\
v_{i} \sin \theta_{i} \\
\omega_{i}
\end{array}\right]
$$

where $\mathbf{x}_{i}(t)=\left[x_{i}, y_{i}, \theta_{i}\right]^{T}$ denotes the state vector in the world frame of the $i$-th robot. $v_{i}$ and $\omega_{i}$ are the linear and angular velocities, respectively.

We first consider path following for each mobile robot in the formation, i.e., we wish to find control law $v_{i}$ and $\omega_{i}$ such that the robot follows a virtual vehicle with position $\mathbf{x}_{d i}=\left[x_{d i}, y_{d i}, \theta_{d i}\right]^{T}$ and inputs $v_{d i}$ and $\omega_{d i}$. A unicycle-type mobile robot is depicted in Fig. 1, together with a spatial path $\Gamma_{i}$ to be followed. The path error with respect to a robot frame is given by

$$
\left[\begin{array}{l}
x_{e i} \\
y_{e i} \\
\theta_{e i}
\end{array}\right]=\left[\begin{array}{ccc}
\cos \theta_{i} & \sin \theta_{i} & 0 \\
-\sin \theta_{i} & \cos \theta_{i} & 0 \\
0 & 0 & 1
\end{array}\right]\left[\begin{array}{l}
x_{d i}-x_{i} \\
y_{d i}-y_{i} \\
\theta_{d i}-\theta_{i}
\end{array}\right] \text {. }
$$

Then, the error dynamics are

$$
\begin{aligned}
& \dot{x}_{e i}=y_{e i} \omega_{i}-v_{i}+\dot{s}_{i} \cos \theta_{e i} \\
& \dot{y}_{e i}=-x_{e i} \omega_{i}+\dot{s}_{i} \sin \theta_{e i} \\
& \dot{\theta}_{e i}=\kappa_{i} \dot{s}_{i}-\omega_{i}
\end{aligned}
$$

where $\kappa_{i}$ is the path curvature and $\dot{s}_{i}$ is the velocity of a virtual vehicle. It is bounded by $0 \leq \dot{s}_{i} \leq \dot{s}_{\max , \mathrm{i}}$.

Next, we consider the coordination problem. To maintain the motion coordination of the whole group, each robot requires an individual parameterized path so that when all path's parameters are synchronized, all robots will be in formation. The velocities at which the mobile robots are required to travel can be handled in many ways. In this paper, there are three velocities to be synchronized, i.e., the velocity $v_{0}$ (or $v_{d i}$ in the robot frame) specifying how fast the whole group of robots should move, the velocity $\dot{s}_{i}$ denoting how fast an individual virtual vehicle moves along the path, and the velocity $v_{i}$ determining how fast an individual real mobile robot travels (see Fig. 2). 

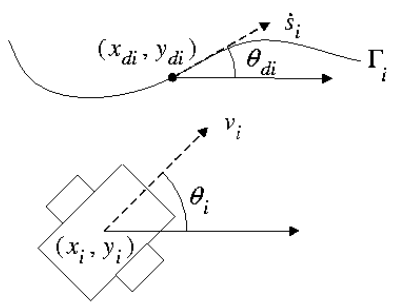

Fig. 1. A graphical representation of a mobile robot and a path.

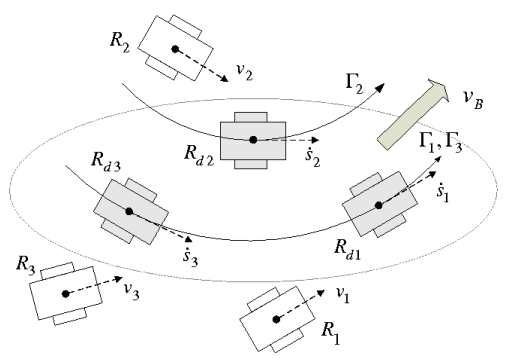

Fig. 2. A graphical representation of coordinated path following.

As well known, the formation graph can be used to describe the relationship among members in the group. It is defined as $\mathcal{G}=(\mathcal{V}, \mathcal{E})$, where $\mathcal{V}=1, \ldots, N$ is the set of robots and $\mathcal{E} \subset \mathcal{V} \times \mathcal{V}$ is the set of relative vectors between robots. Two robots $i$ and $j$ are called neighbors if $(i, j) \in \mathcal{E}$, and the set of neighbors of robot $i$ is denoted by $N_{i} \in \mathcal{V}$. All graphs considered in this paper are undirected and we assume that the undirected $\mathcal{G}$ is connected. In this case, the Laplacian matrix $L$, constructed from $L=D-A$, where the adjacency matrix $A=\left(a_{i j}\right)$ and the diagonal degree matrix $D$, is symmetric positive semi-definite. It has a simple zero eigenvalue and all the other eigenvalues are positive if and only if the graph is connected [12]. This matrix forms the basis for distributed consensus dynamics and captures many properties of the graph (see [12]).

\section{Controller Design}

Define the following variable

$$
\dot{\tilde{s}}_{i}=\dot{s}_{i}-v_{d i},
$$

where $\dot{\tilde{s}}_{i}$ represents the formation speed tracking error of robot $i$. Let us choose

$$
V=\frac{1}{2} \sum_{i=1}^{N}\left(x_{e i}^{2}+y_{e i}^{2}+\frac{1}{k_{4}}\left(\theta_{e i}-\delta_{i}\left(y_{e i}, v\right)\right)^{2}+k_{5} \dot{\tilde{s}}_{i}^{2}+k_{6} \bar{s}_{i}^{2}\right)
$$

as a candidate Lyapunov function, where $k_{4}, k_{5}$, and $k_{6}$ are positive gains. $\bar{s}_{i}=$ $s_{i}-s_{j}-s_{d i j}, j \in N_{i}$ is the coordination error of robot $i$ and $s_{d i j}$ is the desired distance between two neighbors $i$ and $j$. The function $\delta_{i}$ can be interpreted as the desired value for the orientation $\theta_{e i}$ during transients [5]. It is assumed that $\lim _{t \rightarrow \infty} v(t) \neq 0, \delta_{i}(0, v)=0$, and $y_{e i} v \sin \left(y_{e i}\right) \leq 0, \forall y_{e i} \forall v$. The function $\delta_{i}\left(y_{e i}, v\right)$ taken from [6] is $\delta_{i}\left(y_{e i}, v\right)=-\operatorname{sign}\left(v_{d i}\right) \theta_{a} \tanh y_{e i}$ with $\theta_{a}=\frac{\pi}{4}$.

The derivative of $V$ can be computed to give

$$
\dot{V}=\sum_{i=1}^{N}\left[x_{e i} \dot{x}_{e i}+y_{e i} \dot{y}_{e i}+\frac{1}{k_{4}}\left(\theta_{e i}-\delta_{i}\right)\left(\dot{\theta}_{e i}-\dot{\delta}_{i}\right)+k_{5} \dot{\tilde{s}}_{i} \ddot{\tilde{s}}_{i}+k_{6} \bar{s}_{i} \dot{\bar{s}}_{i}\right] .
$$


We first design a controller to stabilize the $x_{e i}, y_{e i}$, and $\theta_{e i}$ dynamics. Substituting (4) into (3), adding $y_{e i} v_{d i} \sin \delta_{i}-y_{e i} v_{d i} \sin \delta_{i}$ to (6), the time derivative along the solutions of (3) yields

$$
\begin{aligned}
\dot{V}= & \sum_{i=1}^{N}\left[x_{e i}\left(y_{e i} \omega_{i}-v_{i}+\left(\dot{\tilde{s}}_{i}+v_{d i}\right) \cos \theta_{e i}\right)+y_{e i}\left(-x_{e i} \omega_{i}+\left(\dot{\tilde{s}}_{i}+v_{d i}\right) \sin \theta_{e i}\right)\right. \\
& \left.+\frac{1}{k_{4}}\left(\theta_{e i}-\delta_{i}\right)\left(\dot{\theta}_{e i}-\dot{\delta}_{i}\right)+y_{e i} v_{d i} \sin \delta_{i}-y_{e i} v_{d i} \sin \delta_{i}+k_{5} \dot{\tilde{s}}_{i} \ddot{\tilde{s}}_{i}+k_{6} \bar{s}_{i} \dot{s}_{i}\right] .
\end{aligned}
$$

Let the control laws for $v_{i}$ and $\omega_{i}$ be defined as

$$
\begin{aligned}
& v_{i}=k_{1} x_{e i}+v_{d i} \cos \theta_{e i} \\
& \omega_{i}=k_{2}\left(\theta_{e i}-\delta_{i}\right)+\omega_{d i}-\dot{\delta}_{i}+k_{4} y_{e i} v_{d i}\left[\frac{\sin \theta_{e i}-\sin \delta_{i}}{\theta_{e i}-\delta_{i}}\right],
\end{aligned}
$$

where $k_{1}$ and $k_{2}$ are positive gains, and $\omega_{d i}=\kappa_{i} v_{d i}$. Then

$$
\begin{aligned}
\dot{V}= & \sum_{i=1}^{N}\left[-k_{1} x_{e i}^{2}-\frac{k_{2}}{k_{4}}\left(\theta_{e i}-\delta_{i}\right)^{2}+x_{e i} \dot{\tilde{s}}_{i} \cos \theta_{e i}+y_{e i} \dot{\tilde{s}}_{i} \sin \theta_{e i}\right. \\
& \left.+\frac{1}{k_{4}}\left(\theta_{e i}-\delta_{i}\right) \kappa_{i} \dot{\tilde{s}}_{i}+y_{e i} v_{d i} \sin \delta_{i}+k_{5} \dot{\tilde{s}}_{i} \ddot{\tilde{s}}_{i}+k_{6} \bar{s}_{i} \dot{\bar{s}}_{i}\right] .
\end{aligned}
$$

To make the derivative of the Lyapunov function $V$ negative, we choose the following consensus controller with a reference velocity

$$
\begin{aligned}
\ddot{s}_{i}= & \dot{v}_{d i}-k_{3}\left(\dot{s}_{i}-v_{d i}\right)-x_{e i} \cos \theta_{e i}-y_{e i} \sin \theta_{e i}-\frac{1}{k_{4}}\left(\theta_{e i}-\delta_{i}\right) \kappa_{i} \\
& -2 k_{5} \sum_{j \in N_{i}}\left(s_{i}-s_{j}-s_{d i j}\right)-k_{6} \sum_{j \in N_{i}}\left(\dot{s}_{i}-\dot{s}_{j}\right)
\end{aligned}
$$

where $k_{3}>0$. Then we can achieve

$$
\dot{V}=\sum_{i=1}^{N}\left(-k_{1} x_{e i}^{2}-k_{2}\left(\theta_{e i}-\delta_{i}\right)^{2}-k_{3}\left(\dot{s}_{i}-v_{d i}\right)^{2}\right)-k_{6} \dot{s}^{T} L \dot{s} \leq 0,
$$

where $\dot{s} \in \mathbb{R}^{N}$ is the stack vector of the robots' path derivative. We now state the main result of the coordinated path-following control for the mobile robots.

Theorem 1. Assume that the undirected formation graph is connected. The control inputs $v_{i}, \omega_{i}$, and $\ddot{s}_{i}$ given in (8), (9), and (11), respectively, for robot $i$ solve the coordinated path-following objective.

Proof. From (12), we have that $\dot{V} \leq 0$, which means that

$$
V \leq V\left(t_{0}\right), \quad \forall t \geq t_{0}
$$


From the definition of $V$, the right hand side of (13) is bounded by a positive constant depending on the initial conditions. Since the left hand side of (13) is bounded, it implies the boundedness of $x_{e i}, y_{e i},\left(\theta_{e i}-\delta_{i}\right), \dot{\tilde{s}}_{i}$ and $\bar{s}_{i}$ for all $t \geq t_{0} \geq 0$. We also assume boundedness of $\dot{s}_{i}$ and $v_{d i}$, implying the boundedness of the the overall closed-loop coordination system on the maximal interval of definition $[0, T)$. This rules out finite escape time so that $T=+\infty$.

From the above argument on the boundedness of $x_{e i}, y_{e i},\left(\theta_{e i}-\delta_{i}\right), \dot{\tilde{s}}_{i}$ and $\bar{s}_{i}$, applying Barbalat's lemma [13] to (12) results in

$$
\lim _{t \rightarrow \infty}\left(x_{e i}, \theta_{e i}-\delta_{i}, \dot{\tilde{s}}_{i}, \dot{\bar{s}}_{i}\right)=0 .
$$

To satisfy path-following tasks, we have to show that $y_{e i}$ converges to zero as $t \rightarrow \infty$. In the closed loop of the $\theta_{e i}$ dynamics

$$
\dot{\theta}_{e i}=\kappa_{i} \dot{\tilde{s}}_{i}-k_{2}\left(\theta_{e i}-\delta_{i}\right)-y_{e i} v_{d i}\left[\frac{\sin \theta_{e i}-\sin \delta_{i}}{\theta_{e i}-\delta_{i}}\right],
$$

we can conclude that $\lim _{t \rightarrow \infty}\left(y_{e i}\right)=0$ since $\lim _{t \rightarrow \infty}\left(\theta_{e i}-\delta_{i}, \dot{\tilde{s}}_{i}\right)=0$ and $v_{d i}$ does not converge to zero.

Since $L$ is positive semidefinite, it follows that $L \dot{s}=0 . L$ has a single zero eigenvalue with corresponding eigenvector $\overrightarrow{\mathbf{1}}$. It follows that $\dot{s}$ belongs to $\operatorname{span}\{\overrightarrow{\mathbf{1}}\}$. Hence $\dot{s}_{i}=\dot{s}_{j}, \forall i, j \in N$, and $\dot{s}_{i}$ converges to $v_{d i}$, which in turn, implies that $\ddot{s}_{i}=\dot{v}_{d i}$. From this fact, we can get

$$
\lim _{t \rightarrow \infty}\left(s_{i}-s_{j}-s_{d i j}\right)=0 .
$$

Define $s_{d i j}=s_{d i}-s_{d j}$, where $s_{d i}$ and $s_{d j}$ are the path's desired parameters of robot $i$ and robot $j$, respectively. We then have $s_{i}-s_{j}-s_{d i j}=s_{i}-s_{j}-\left(s_{d i}-s_{d j}\right)=$ $\left(s_{i}-s_{d i}\right)-\left(s_{j}-s_{d j}\right)=\hat{s}_{i}-\hat{s}_{j}$. Then we obtain $L s+s_{d}=0 \Rightarrow L \hat{s}=0$. Thus, all $\hat{s}_{i}$ are equal to a common value, i.e., $s_{i}-s_{j}=s_{d i j}, j \in N_{i}, \forall i, j$. We conclude that the robots converge to the desired configuration.

\section{Results}

Simulations and real-world experiments of the control system as established in the previous section were carried out to evaluate the performance of our control law. Regarding more realistic situations in robot motions, we took into account the maximum velocities: $\left|v_{i}\right| \leq 0.5 \mathrm{~m} / \mathrm{s},\left|\omega_{i}\right| \leq 1.0 \mathrm{rad} / \mathrm{s}$. We performed a velocity scaling given in [14] so as to preserve the curvature radius corresponding to the nominal velocities. The control gains were set to $k_{1}=0.25, k_{2}=1.0$, $k_{3}=0.2, k_{4}=5.0, k_{5}=0.5, k_{6}=0.2$ and the desired speed for the whole group of robots was $v_{0}=0.2 \mathrm{~m} / \mathrm{s}$.

\subsection{Simulations}

Six mobile robots were required to follow a lemniscate curve given by

$$
x_{d}(t)=\frac{2.3 \cos \theta_{d}(t)}{1+\sin ^{2} \theta_{d}(t)}, y_{d}(t)=\frac{2.3 \sin \theta_{d}(t) \cos \theta_{d}(t)}{1+\sin ^{2} \theta_{d}(t)},
$$


and to maintain a desired formation described by the following elements of the adjacency matrix: $a_{14}=a_{13}=a_{36}=a_{56}=a_{25}=1$. The superimposed snapshots are shown in Fig. 3 and the coordination errors converging to zero are seen in Fig. 4. The velocity tracking errors and the path errors of each robot also converge to zero, satisfying the path-following objective. Due to lack of space, those errors are not shown in this paper.

\subsection{Real-World Experiments}

The mobile robots, shown in Fig. 5, were used in real-world experiments in this paper. The robot controller is an ATMEGA644 microprocessor with $64 \mathrm{~KB}$ flash program memory, $16 \mathrm{MHz}$ clock frequency and $4 \mathrm{~KB}$ SRAM. The robot orientation was measured by a Devantech CMPS03 compass. The localization was given by a camera looking down upon the robot's workplace and a PC was used to compute the control inputs and then sent these inputs to the robot via WLAN. The lemniscate curve similar to the path in the simulation was employed in the first experiment. Each robot was required to maintain a column formation described by $s_{d 12}=s_{d 23}=75 \mathrm{~cm}$. The elements $a_{12}=a_{21}=1, a_{23}=a_{32}=1$ in the adjacency matrix represented the information exchange in the formation graph. The experimental results are plotted in Fig. 6. As seen in Fig. 7, the coordination tasks are satisfied. The coordination errors are less than $10 \mathrm{~cm}$ and the virtual vehicle of each robot can travel at the desired speed $v_{0}=0.2 \mathrm{~m} / \mathrm{s}$. Likewise, the path-following tasks are attained as seen in Fig. 8. In the second experiment, each robot followed its own path, i.e., a sinusoidal curve for robot 1 and robot 3 , and a straight line for robot $2 . s_{d i j}$ was set to 0 and the elements of the adjacency matrix were set to $a_{12}=a_{21}=1, a_{23}=a_{32}=1$. The results are depicted in Fig. 9. The coordination errors and the velocity of each virtual vehicle are seen in Fig. 10. The experimental results show the effectiveness of our proposed control law: the group of robots can travel at the desired speed $v_{0}$ while keeping a desired formation. The main sources of disturbances during

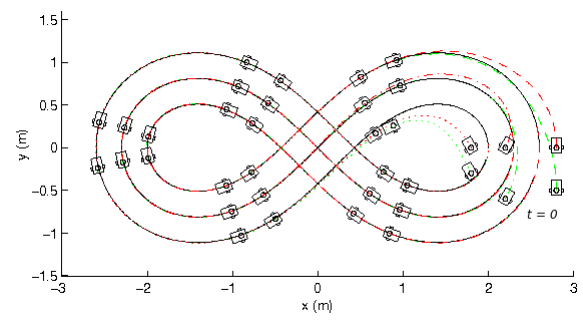

Fig. 3. Simulation: the superimposed snapshots.
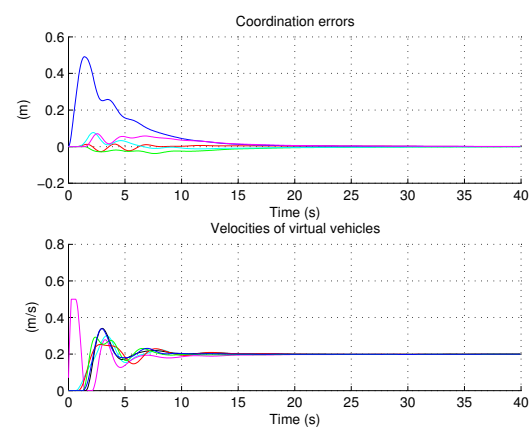

Fig. 4. Simulation: the coordination errors and the velocities of virtual vehicles. 


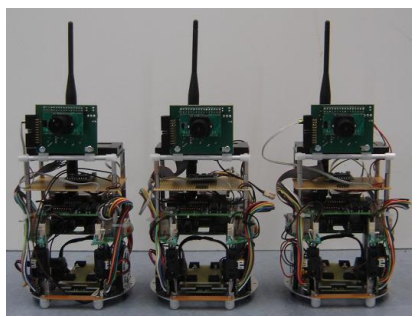

Fig. 5. The mobile robots $(12 \mathrm{~cm}$ diameter) used in experiments.
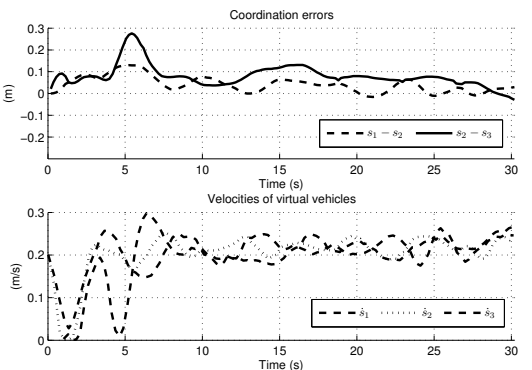

Fig. 7. Exp. 1: the coordination errors and the velocities of virtual vehicles.

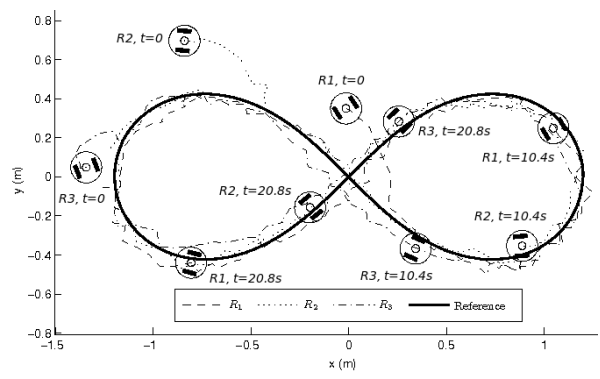

Fig. 6. Exp. 1: the superimposed snapshots at $\mathrm{t}=0 \mathrm{~s}, \mathrm{t}=10.4 \mathrm{~s}$, and $\mathrm{t}=20.8 \mathrm{~s}$.

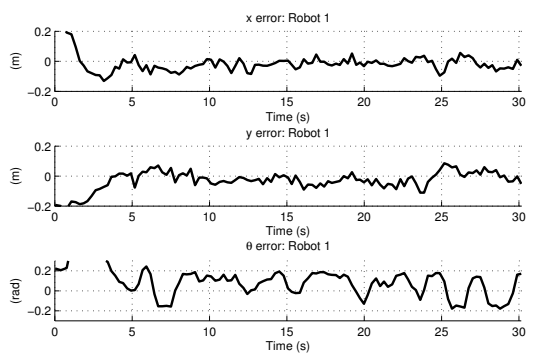

Fig. 8. Exp. 1: the position errors of robot 1 .

experiments include sensor distortion, vision-system delays, and communication delays.

\section{Conclusions and Future Work}

In this paper, we developed a new control law for coordinated path following of mobile robots. Each mobile robot can be steered along a set of given spatial paths, while it can keep a desired inter-vehicle coordination pattern. The solution adopted for coordinated path following built on Lyapunov function techniques and consensus algorithms. The desired formation pattern was achieved by controlling the path derivative such that the coordination error converges to zero.

Future research includes the extension of our results to robots in more complex environments. For example, communication can introduce time-delays in signal propagation among members, the information exchange topology is not necessarily fixed, and obstacles may appear in the robot's path.

\section{References}

1. Murray, R. M.: Recent research in cooperative-control of multivehicle systems. Journal of Dynamics, Systems, Measurement and Control, 129(5), pp. 571-583, 2007 


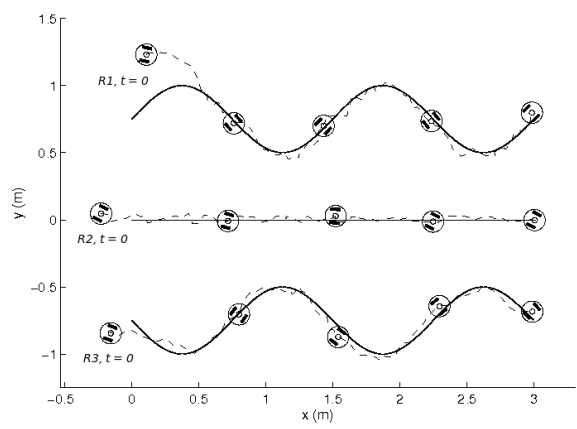

Fig. 9. Exp. 2: the superimposed snapshots at $\mathrm{t}=0 \mathrm{~s}, \mathrm{t}=5.2 \mathrm{~s}, \mathrm{t}=10.6 \mathrm{~s}$, $\mathrm{t}=16.2 \mathrm{~s}$, and $\mathrm{t}=21.6 \mathrm{~s}$.

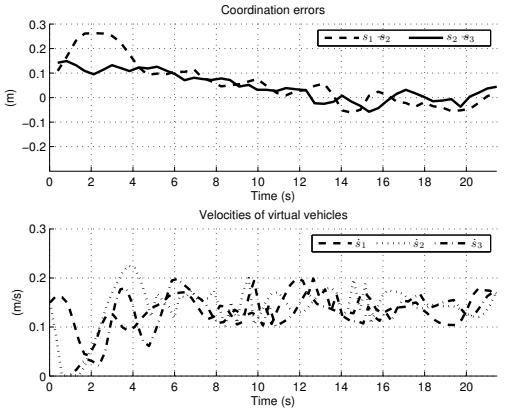

Fig. 10. Exp. 2: the coordination errors and the velocities of virtual vehicles $\left(v_{0}=0.15 \mathrm{~m} / \mathrm{s}\right)$.

2. Das, A. K., Fierro, R., Kumar, V., Ostrowski, J. P., Spletzer, J., and Taylor, C. J.: A vision-based formation control framework. IEEE Trans. on Robotics and Automation, 18(5), pp. 813-825, Oct. 2002

3. Lewis, M. A. and Tan, K. -H.: High precision formation control of mobile robots using virtual structures. Autonomous Robots, 4(4), pp. 387-403, 1997

4. Balch, T. and Arkin, R. C.: Behavior-based formation control for multirobot teams. IEEE Trans. on Robotics and Automation, 14(6), pp. 1-15, 1998

5. Micaelli, A. and Samson, C.: Trajectory-tracking for unicycle-type and two-steeringwheels mobile robots. Technical Report No. 2097, INRIA, Sophia-Antipolis, 1993

6. Soeanto, D., Lapierre, L. and Pascoal, A.: Adaptive non-singular path-following, control of dynamic wheeled robots. Proc. of Int. Conf. on Advanced Robotics, Coimbra, Portugal, pp. 1387-1392, 2003

7. Egerstedt, M., Hu, X., and Stotsky, A.: Control of mobile platforms using a virtual vehicle approach. IEEE Trans. on Automatic Control, 46(11), pp. 1777-1782, 2001

8. Ihle, I. A. F., Arcak, M., and Fossen, T. I.: Passivity-based designs for synchronized path-following. Automatica, 43(9), pp. 1508-1518, 2007

9. Ghabcheloo, R., Aguiar, A., Pascoal, A., Silvestre, C., Kaminer, I., and Hespanha, J.: Coordinated path-following in the presence of communication losses and time delays. SIAM - Journal on Control and Optimization, 48(1), pp. 234-265, 2009

10. Ghabcheloo, R., Pascoal, A., Silvestre, C., and Kaminer, I.: Nonlinear coordinated path following control of multiple wheeled robots with bidirectional communication constraints. Int. Journal of Adaptive Control and Signal Processing, 20, pp. 133-157, 2007

11. Ghomman, J., Saad, M., and Mnif, F.: Formation path following control of unicycletype mobile robots. Proc. of IEEE Conf. on Robotics and Automation, Pasadena, CA, pp. 1966-1972, 2008

12. Fax, J. A. and Murray, R. M.: Graph laplacians and vehicle formation stabilization. Proc. of the 15th IFAC World Congress, Barcelona, Spain, 2002

13. Khalil, H. K.: Nonlinear Systems. Prentice Hall, 2002

14. Oriolo, G., De Luca, A., and Vendittelli, M.: WMR control via dynamic feedback linearization: design, implementation and experimental validation. IEEE Trans. on Control Systems Technology, 10(6), pp. 835-852, 2002 